Case Report

\title{
A Silent and Very Early Post-Liver Transplant Death by Candida Arteritis
}

Kyungmin Ko ${ }^{1, *}$, Juan F. Guerra ${ }^{2}$, David F. Garvin ${ }^{1}$

1. Department of Pathology, MedStar Georgetown University Hospital, 3900 Reservoir Rd NW, Washington, DC 20007, USA; E-Mails: Kyungmin.Ko@gunet.georgetown.edu; david.garvin3@verizon.net

2. Medstar Georgetown Transplant Institute, MedStar Georgetown University Hospital, 3800 Reservoir Rd NW, 2PHC Washington, DC 20007, USA; E-Mail: Juan.Guerra@gunet.georgetown.edu

* Correspondence: Kyungmin Ko; E-Mail: Kyungmin.Ko@gunet.georgetown.edu

Academic Editor: Yasuhiko Sugawara

Special Issue: Infections in Liver Transplantation

OBM Transplantation

2019, volume 3 , issue 4

doi:10.21926/obm.transplant.1904089
Received: August 29, 2019

Accepted: October 24, 2019

Published: October 28, 2019

\begin{abstract}
Background: Graft site candidiasis is a serious complication after solid organ transplantation, often presenting as invasive arteritis leading to graft loss or even death.

Case Report: We report a case of anastomotic site candidiasis leading to arterial rupture and death at postoperative day 10 with no clinical warning signs. Preservation fluid cultures were negative and postoperative Doppler ultrasonography did not show vascular compromise. Postmortem examination revealed microscopic arterial wall abscesses harbouring hyphae and yeast consistent with Candida species.

Conclusions: Our case demonstrates a particularly early and silent death post-liver transplant due to Candida arteritis and highlights the limitations of current preventive and screening measures. We discuss potential routes of contamination and control measures.
\end{abstract}

\section{Keywords}

Candida arteritis; post-liver transplant candidiasis; invasive candidiasis

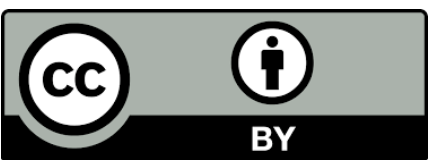

(C) 2019 by the author. This is an open access article distributed under the conditions of the Creative Commons by Attribution License, which permits unrestricted use, distribution, and reproduction in any medium or format, provided the original work is correctly cited. 


\section{Introduction}

Fungal infection is a well-known, leading cause of morbidity and mortality in patients with solid organ transplantation (SOT). Candida is the most common fungal pathogen after orthotopic liver transplantation (OLT), and the most frequent cause of invasive fungal infections [1]. When it infects the liver transplant recipient, it presents most commonly as candidemia, and less commonly as peritonitis, abdominal abscess, or biliary tract infection [2]. In rare cases it presents as arteritis.

Candida arteritis is a devastating form of candidiasis. In SOT recipients this generally occurs as anastomotic site infection of graft vessels [3-7], often leading to rupture or mycotic aneurysm, and subsequent graft loss. Here we report a case of invasive Candida arteritis leading to massive abdominal hemorrhage and death at postoperative day (POD) 10 without any clinical suspicion. This case, to our knowledge, represents the earliest reported post-OLT mortality resulting from proven invasive candidiasis, and highlights the limitation of current detection methods.

\section{Case Report}

The donor was an ABO-compatible 39-year-old man who died of anoxic brain injury secondary to drug use. The donor did not meet CDC criteria to be considered as a high-risk donor. Liver function tests (LFTs) were within normal limits at the time of procurement. Pre-transplant biopsy of the donor liver showed only minimal chronic inflammation, and no macrovesicular or microvesicular steatosis. Donor antibiotic history included piperacillin/tazobactam and linezolid for a presumed aspiration pneumonia. There was no known gastrointestinal tract defect, either before or during procurement. Blood and urine cultures were negative. Sputum gram stain showed heavy growth yeast, not Cryptococcus species. Standard donor infectious diseases screening and testing (including HIV, HBV, HCV, CMV, EBV, Toxoplasma, and syphilis) were performed, and the only positive result was EBV (IgG).

The recipient was a 58-year-old man with alcoholic cirrhosis complicated with a welldifferentiated hepatocellular carcinoma that measured $2.2 \mathrm{~cm}$ on contrast-enhanced MRI, which was treated with transcatheter arterial chemoembolization 8 months prior, with no metastatic disease. His MELD laboratory score was 9 based on the following parameters: serum creatinine $1.72 \mathrm{mg} / \mathrm{dL}$, bilirubin $1.4 \mathrm{mg} / \mathrm{dL}$, INR 1.1, and serum sodium $138 \mathrm{mEq} / \mathrm{L}$. At the time of transplant, he denied any infectious issues. Based on his cancer history, the Regional Review Board approved a MELD exception score of 28 for transplant prioritization purposes, in accordance with United Network for Organ Sharing (UNOS) protocols.

UW (University of Wisconsin) solution was used as the preservation fluid. The operation was performed under standard aseptic condition, with no massive intraoperative contamination. The suprahepatic and infrahepatic venae cavae were anastomosed in an end-to-end fashion. The donor had a replaced left hepatic artery that was left intact from its origin at the celiac trunk, and the donor celiac artery was anastomosed to the recipient common hepatic artery in an end-to-end fashion. Because thrill at the left hepatic artery was weak, the stump of the splenic artery was used to perform an arterial reconstruction. Arterial intrahepatic flow was confirmed 
intraoperatively using a Doppler. Perfect anastomotic hemostasis was evident at the end of the case. The donor and recipient ducts matched in size and were anastomosed in end-to-end fashion. Two \#10 Jackson-Pratt drains were placed: one behind the right lobe of the liver, one behind the biliary anastomosis.

The patient received a standard immunosuppressive protocol including glucocorticoids, tacrolimus, and mycophenolate mofetil. Intact vascular flow and absence of focal lesions was demonstrated on postoperative day two by ultrasound. The hepatic graft demonstrated good function, with postoperative LFTs down-trending on subsequent days. He was intermittently transfused with a total of 6 units of Red Blood Cells as needed throughout his postoperative course. Aspirin and subcutaneous heparin were given due to the complex hepatic artery reconstruction and DVT prophylaxis, and platelet count and coagulation times remained stable. Prophylactic antibiotic treatment was administered with ciprofloxacin and metronidazole for five days. He was extubated on day two and tolerated oral feeding from day three. Preservation fluid culture result was negative. The patient remained afebrile and normotensive throughout his hospital course, and no postoperative bacterial culture, fungal culture, or beta-D-glucan assay was ordered.

On postoperative day ten, after an uneventful recovery period with the patient being ready to be discharged, he reported presyncopal symptoms and immediately became unresponsive, rapidly progressing to circulatory collapse and death, despite prolonged cardiopulmonary resuscitation.

On postmortem examination, $3.15 \mathrm{~L}$ of blood and clot was found in the abdominal cavity. Meticulous microscopic examination revealed no anastomotic dehiscence of the vascular anastomoses. Instead, abundant acute inflammatory infiltrates and abscesses on the tissue surrounding hepatic arteries were seen. Gomori-Methenamine-Silver (GMS) and tissue Gram stain revealed numerous pseudohyphae morphologically consistent with Candida forming arterial wall abscesses (Figure 1). Candida albicans and glabrata were isolated from postmortem blood culture.

\section{Discussion}

The current case demonstrates a fatality from rapid progression of post-OLT anastomotic Candida arteritis that was discovered upon postmortem examination.

Graft site candidiasis is a distinct form of posttransplant candidiasis. Candida infections in OLT recipients most commonly present as candidemia, and less commonly as peritonitis, abdominal abscess, or biliary tract infection [2]. In contrast, arteritis is the main form of Candida occurring at the graft site. In a review of 25 graft-site candidiasis cases after kidney transplantation, the majority (14 cases) were renal arteritis [5]. Graft site candidiasis is also associated with PF culture positivity [3, 5-7]. In a review of 28 liver transplant patients with yeast culture-positive PF, 28.6\% presented with graft site arteritis [7]. Graft-site candidiasis occurs earlier compared with transplant fungal infections. The median interval to developing candidiasis post-liver transplant is 151 days with an interquartile range of 14 to 1037 days [2]. Cases reported in reviews of liver preservation fluid (PF) culture positivity [7-9], or case reports $[4,6,10,11]$ show an earlier onset.

The point of Candida entry determines where to direct control measures. The organism may be transmitted from the donor, introduced during donor organ procurement, or introduced during transplantation. For individual cases, the source of Candida is usually indeterminate. Several observations show that Candida most often contaminates the graft prior to transplantation. There 
are cases where multiple recipients from the same donor were affected $[4,5,12]$. The association between PF culture positivity and graft-site Candidiasis also supports this [3, 5-7]. In some of these cases, the genotypes of the Candida in different recipients were confirmed to be identical to the genotype of Candida found in the donor or PF $[5,6]$.

\subsection{Donor Screening and Testing}

Donor screening and testing is the principal means of preventing donor-derived infections. The screening process keeps true donor-derived transmission rare. The Organ Procurement and Transplantation Network (OPTN) ad hoc Disease Transmission Advisory Committee (DTAC) has recommended considering any early posttransplant disease as potentially of donor origin [13]. In the United States, a potential donor-derived transmission of infectious disease is reported to the OPTN in less than one percent of donors, and many of these are not confirmed disease transmissions [13]. Fungal infections make up only $15 \%$ of this fraction. Most of the time, donors with fungal sepsis are screened out, since the condition must be communicated to the transplant center prior to transplantation [14]. Also, OPTN Policies require that donor blood culture is performed, and results reported to the receiving transplant center.

However, localized infection in the organ or introduction of the organism during procurement would evade donor screening and testing. It is thought that introduction during procurement is more likely than true donor transmission $[12,15]$. Some suggest that contamination from the donor's own gut microbiota occurs during procurement $[5,12]$, especially when there is donor antibiotic use, prolonged ICU stay, or trauma to the gastrointestinal tract. In the current case, the heavy colonization of the respiratory system in the setting of broad-spectrum antibiotics is a source of contamination during procurement. Communication of risk factors alerts the recipient center of the possibility of infection, but these risk factors are very common and nonspecific. More evidence is needed to define the donor or procurement risk factors and to develop evidencebased recommendations.

\subsection{Preservation Fluid Culture}

PF culture is an indirect test for organisms present on the harvested organ. Unlike donor or procurement risk factors, positivity is a clear indication for antifungal therapy. The frequency of PF contamination by Candida vary between institutions and ranges from $3 \%-9 \%$ in kidney transplantation [16-18], and $0.4-4.1 \%$ in liver transplantation $[8,9,18]$. When compared within the same institution using a standardized microbiological protocol, PF contamination in liver transplantation (4.1\%) seemed to be slightly higher than that in kidney transplantation (3.1\%) [18]. The rate of complications among patients with PF culture-positive Candida appears to be smaller for liver transplant patients than for kidney transplant patients [7]. Neither the American Society of Transplantation (AST) nor the European Society of Clinical Microbiology and Infectious Diseases (ESCMID) has found current evidence to be enough to recommend routine culture of preservation fluid [19, 20], and OPTN Policies do not mandate preservation fluid culture [21]. In contrast, routine preservation fluid culture is strongly recommended in France by the Agence de la Biomédecine [22], and it is routinely performed in our institution. To our knowledge there is no reported sensitivity of PF culture for post-liver transplant candidiasis. It is reasonable to hypothesize that both the sensitivity of PF culture and rapidity of onset correlates with inoculum 
size. If so, the early onset and PF culture negativity of our case would suggest contamination during the transplantation or shortly thereafter with a sizeable inoculum.

PF has been implicated as the mediator of infection, but PF culture positivity by itself does not implicate PF as a mediator of infection [10]. Instead, positivity may result from contact with organisms already on the graft. In our case, the Candida appeared to be more in continuity with the outer aspect of the arterial wall, as if it was invading inward from the adventitia, contrary to what would be expected if infection began intraluminally from the preservation fluid (Figure 1). Even in PF culture-positive cases, candidiasis predominantly localizes to the arterial anastomosis site [7]. This appears more consistent with local contamination rather than infection mediated by perfused fluid.

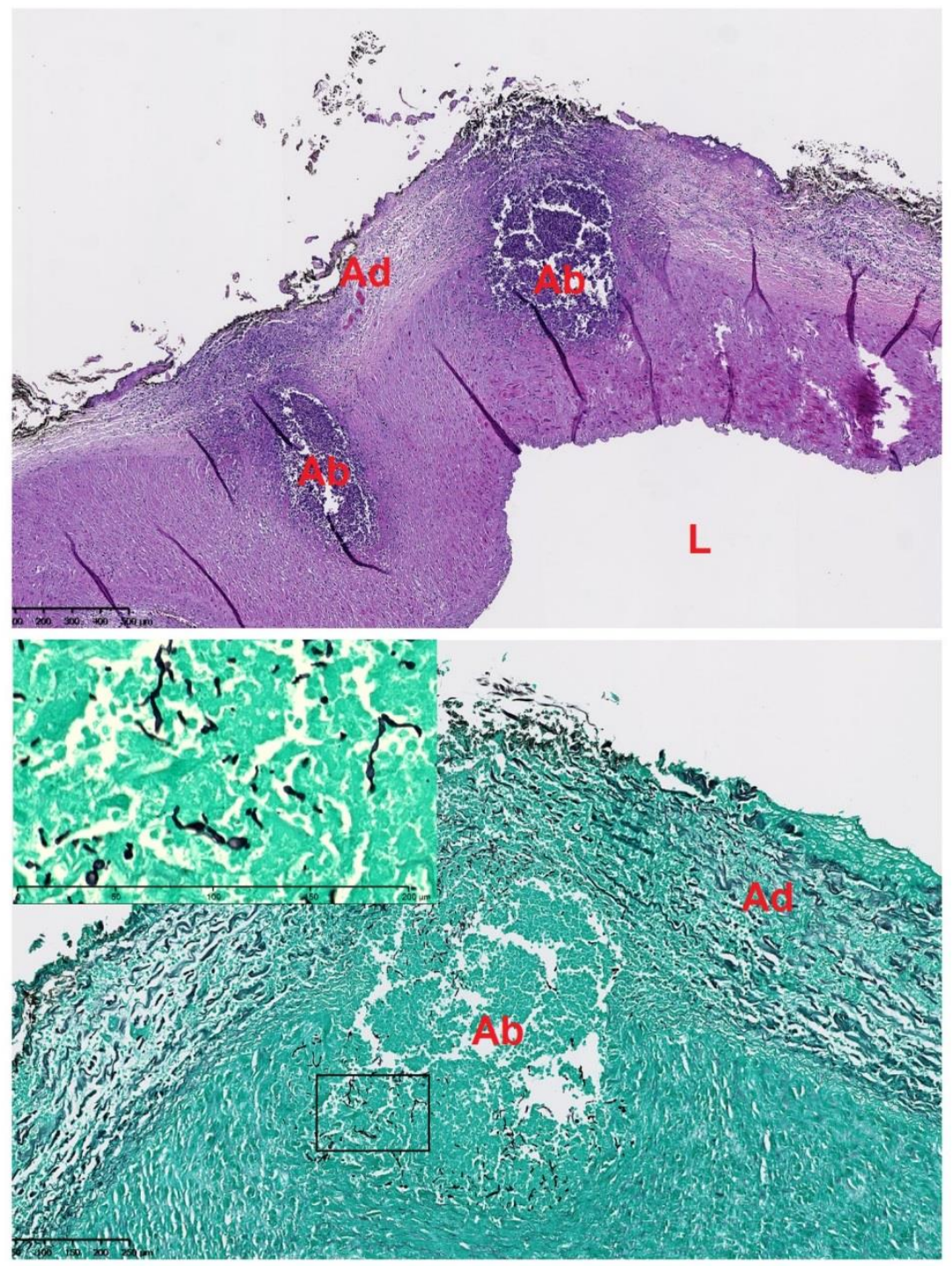

Figure 1 Photomicrographs of arterial invasion by Candida. Top: Arterial wall abscess (Ab) near anastomosis site, H\&E. The abscess involves the adventitia (Ad) and media. It disrupts the external elastic lamina and adventitial connective tissue. Bottom: GomoriMethenamine-Silver stain, showing pseudohyphae and budding yeast consistent with Candida species (inset). There is nonspecific staining in the adventitia. Ab: abscess, Ad: adventitia, L: arterial lumen. 


\subsection{Recipient Monitoring}

With no reason for suspicion, we did not treat the patient for Candida, nor did we perform surgical intervention. This calls into question whether we should consider routine blood culture or additional diagnostic modalities for monitoring of transplant recipients. For example, the 1,3- $\beta$-Dglucan (BDG) assay was found to be a good biomarker to rule out invasive fungal infection [23]. The ESCMID recommends BDG quantification in patients with risk factors or symptoms [24]. Also, radiologic monitoring is important for detecting aneurysm or rupture and timely surgical intervention [7]. Considering the unremarkable Doppler ultrasound in our case, abrupt presentation at a moment of postural change, and brief presyncopal symptom, it is possible that a defect formed abruptly in an already weakened arterial wall. Closer radiologic monitoring of recipients may enable detection of silent pathological processes [7]. It may also be worth exploring imaging techniques for probing structural weakness in the arterial wall before it bleeds.

\section{Conclusions}

In conclusion, the current case demonstrates that graft site Candida arteritis can be more insidious and catastrophic than expected and highlights the limitation of donor screening, preservation fluid culture, and Doppler ultrasound as a means of detection. Other tests such as routine blood cultures, or beta-D-glucan assay, or improvement in imaging techniques may deserve consideration for monitoring liver transplant recipients.

\section{Author Contributions}

Kyungmin Ko prepared the initial manuscript. Juan F. Guerra and David F. Garvin edited the manuscript. Kyungmin Ko and David F. Garvin performed the postmortem examination. All three authors reviewed medical records and literature.

\section{Competing Interests}

The authors have declared that no competing interests exist.

\section{References}

1. Hernandez MDP, Martin P, Simkins J. Infectious complications after liver transplantation. Gastroenterol Hepatol. 2015; 11: 741-753.

2. Bassetti M, Peghin M, Carnelutti A, Righi E, Merelli M, Ansaldi F, et al. Invasive Candida infections in liver transplant recipients: Clinical features and risk factors for mortality. Transplant Direct. 2017; 3: e156.

3. Mai H, Champion L, Ouali N, Hertig A, Peraldi MN, Glotz D, et al. Candida albicans arteritis transmitted by conservative liquid after renal transplantation: A report of four cases and review of the literature. Transplantation. 2006; 82: 1163-1167.

4. El-Bandar N, Kroy DC, Fuller TF, Kramer J, Liefeldt L, Budde K, et al. Development of graft-site Candidiasis in 3 solid organ transplant recipients from the same donor. Am J Case Rep. 2017; 18: 777-781. 
5. Albano L, Bretagne S, Mamzer-Bruneel M, Kacso I, Desnos-Ollivier M, Guerrini P, et al. Evidence that graft-site Candidiasis after kidney transplantation is acquired during organ recovery: A multicenter study in France. Clin Infect Dis. 2009; 48: 194-202.

6. Addeo P, Saouli AC, Woehl-Jaegle ML, Ellero B, Oussoultzoglou E, Marcellin L, et al. Candida albicans arteritis transmitted by preservation fluid after liver transplantation. Ann Transplant. 2014; 19: 64-67.

7. Levesque E, Paugam-Burtz C, Saliba F, Khoy-Ear L, Merle J, Jung B, et al. Fungal complications after Candida preservation fluid contamination in liver transplant recipients. Transpl Int. 2015; 28: 1308-1316.

8. Krawczyk M. Incidence, pattern and clinical relevance of microbial contamination of preservation fluid in liver transplantation. Ann Transplant. 2012; 17: 20-28.

9. Janny S, Bert F, Dondero F, Durand F, Guerrini P, Merckx P, et al. Microbiological findings of culture-positive preservation fluid in liver transplantation. Transpl Infect Dis. 2011; 13: 9-14.

10. Levesque E, Suet G, Merle JC, Compagnon P, Amathieu R, Feray C, et al. Candida vascular complication in a liver transplant recipient due to yeast contamination of preservation solution. Transpl Infect Dis. 2014; 16: 827-829.

11. Llado L, Sole C, Bodro M, Baliellas C, Sabe N, Petit A, et al. Candida arteritis occurring in a liver transplant recipient. Transpl Infect Dis. 2014; 16: 465-468.

12. Calviño J, Romero R, Pintos E, Novoa D, Mardaras J, Arcocha V, et al. Renal artery rupture secondary to pretransplantation Candida contamination of the graft in two different recipients. Am J Kidney Dis. 1999; 33: E1-E5.

13. Ison MG, Nalesnik MA. An update on donor-derived disease transmission in organ transplantation. Am J Transplant. 2011; 11: 1123-1130.

14. Ison MG, Hager J, Blumberg E, Burdick J, Carney K, Cutler J, et al. Donor-derived disease transmission events in the United States: Data reviewed by the OPTN/UNOS Disease Transmission Advisory Committee. Am J Transplant. 2009; 9: 1929-1935.

15. Gottesdiener KM. Transplanted infections: Donor-to-host transmission with the allograft. Ann Intern Med. 1989; 110: 1001-1016.

16. Rodrigues BF, Natário AS, Vizinho RS, Jorge CM, Weigert AL, Martinho A, et al. Candida species contamination of preservation fluid outcome of renal transplantation in 6 patients. Transplant Proc. 2013; 6: 2215-2219.

17. Ranghino A, Diena $D$, Simonato $F$, Messina $M$, Burdese $M$, Piraina $V$, et al. Clinical impact of bacterial contamination of perfusion fluid in kidney transplantation. SpringerPlus. 2016; 5: 7.

18. Botterel F, Foulet F, Legrand P, Soria A-, Farrugia C, Grimbert P, et al. Yeast contamination of kidney, liver and cardiac preservation solutions before graft: Need for standardisation of microbial evaluation. J Hosp Infect. 2010; 76: 52-55.19. Organ Procurement and Transplantation Network Policies. 2019 Sep 24.

19. Singh N, Huprikar S, Burdette SD, Morris MI, Blair JE, Wheat LJ, et al. Donor-derived fungal infections in organ transplant recipients: Guidelines of the American Society of Transplantation, Infectious Diseases Community of Practicet. Am J Transplant. 2012; 12: 2414-2428.

20. Len O, Garzoni C, Lumbreras C, Molina I, Meije Y, Pahissa A, et al. Recommendations for screening of donor and recipient prior to solid organ transplantation and to minimize transmission of donor-derived infections. Clin Microbiol Infect. 2014; 20: 10-18. 
21. Organ Procurement and Transplantation Network Policies. 2019 Sep 24.

22. Prevention de la transmission de bacteries et d'agents fongiques aux receveurs d'organes. Médecine et Maladies Infectieuses. 2009; 39: 674.

23. Levesque E, Rizk F, Noorah Z, Aït-Ammar N, Cordonnier-Jourdin C, El Anbassi S, et al. Detection of (1,3)- $\beta$-d-Glucan for the diagnosis of invasive fungal infection in liver transplant recipients. Int J Mol Sci. 2017; 18. doi: 10.3390/ijms18040862.

24. Gavaldà J, Meije Y, Fortún J, Roilides E, Saliba F, Lortholary O, et al. Invasive fungal infections in solid organ transplant recipients. Clin Microbiol Infect. 2014; 20: 27-48.

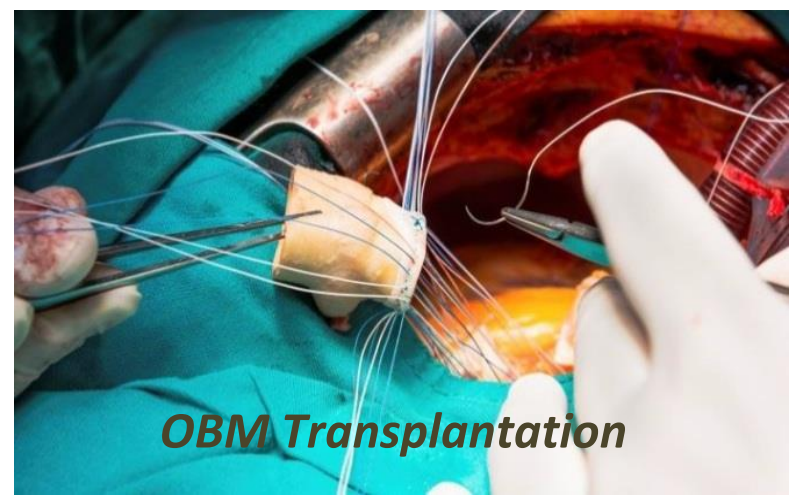

Enjoy OBM Transplantation by:

1. Submitting a manuscript

2. Joining in volunteer reviewer bank

3. Joining Editorial Board

4. Guest editing a special issue

For more details, please visit: http://www.lidsen.com/journals/transplantation 